\title{
Elevated exhaled acetone concentration in stage $C$ heart failure patients with diabetes mellitus
}

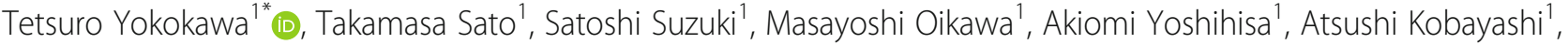 \\ Takayoshi Yamaki ${ }^{1}$, Hiroyuki Kunii ${ }^{1}$, Kazuhiko Nakazato ${ }^{1}$, Hitoshi Suzuki ${ }^{1}$, Shu-ichi Saitoh', Takafumi Ishida', \\ Akito Shimouchi $^{2}$ and Yasuchika Takeishi ${ }^{1}$
}

\begin{abstract}
Background: Breath acetone is a noninvasive biomarker of heart failure; however, its significance in heart failure patients with diabetes mellitus has yet to be clarified. The objective of this study is to investigate whether exhaled acetone concentration is a noninvasive biomarker in heart failure patients with diabetes mellitus.

Methods: This study prospectively included 35 diabetic patients with stage $C$ heart failure and 20 diabetic patients with or at risk of heart failure (stage A or B). Exhaled breath was collected after an overnight fast.

Results: The stage $C$ group had significantly higher brain natriuretic peptide levels, larger left ventricular diameter, lower left ventricular ejection fraction, and more frequent use of $\beta$-blocker, compared with the stage A or B group. The stage $C$ group had higher exhaled acetone concentrations than the stage $\mathrm{A}$ or $\mathrm{B}$ group $(p=0.013)$. Exhaled acetone concentration was correlated with total ketone bodies $(r=0.588, p<0.001)$ and brain natriuretic peptide $(r=0.415, p=0.002)$.
\end{abstract}

Conclusion: Stage $C$ heart failure patients with diabetes mellitus have elevated exhaled acetone concentrations. Exhaled acetone concentration could be a noninvasive biomarker in heart failure patients with diabetes mellitus.

Keywords: Acetone, Breath analysis, Heart failure, Diabetes mellitus

\section{Background}

Energy metabolism mainly depends on fatty acids and glucose in the heart [1]. However, the failing heart relies on ketone bodies, which are minor substrates for the normal myocardium [2, 3]. In addition, ketone body utilization is impaired in the skeletal muscle, as systemic deconditioning in heart failure [4]. Elevated myocardial energy expenditure is associated with significant changes of 3-hydroxybutyrate and acetone, which are ketone bodies [5]. These altered cardiac and systemic metabolism causes elevated blood ketone body levels in heart failure patients [6].

Acetone, a volatile component of ketone bodies, is detected in the breath. Breath acetone concentration is

\footnotetext{
* Correspondence: yokotetu@fmu.ac.jp

${ }^{1}$ Department of Cardiovascular Medicine, Fukushima Medical University, 1

Hikarigaoka, Fukushima, Fukushima 960-1295, Japan

Full list of author information is available at the end of the article
}

elevated in heart failure, following the elevation of blood ketone body levels, and has been reported to be a noninvasive biomarker of heart failure [7, 8]. Exhaled acetone might also be detected in patients with diabetes mellitus, lung cancer, and allergic asthma [9-11].

Among the diseases related to exhaled acetone, diabetes mellitus is a representative cause of ketosis, and is an important comorbidity in heart failure patients $[12,13]$. As far as we know, only one report described exhaled acetone in a heterogeneous group with heart failure, including some diabetes mellitus patients [14]. Exhaled acetone concentration has not been investigated in diabetic patients with heart failure only. We investigated whether exhaled acetone concentration could be a noninvasive biomarker in heart failure patients with diabetes mellitus. 


\section{Methods}

This cross sectional study included 35 patients with stage $\mathrm{C}$ heart failure and diabetes mellitus (stage $\mathrm{C}$ group) who were hospitalized for 6 months between September 2016 and March 2017 in Fukushima Medical University Hospital [15]. Diagnosis of heart failure was evaluated by two or more independent cardiologists with two major criteria or one major criterion in conjunction with two minor Framingham criteria [16]. Heart failure was categorized in subtypes $\mathrm{A}, \mathrm{B}$ and $\mathrm{C}$ according to the American College of Cardiology Foundation/American Heart Association guidelines [15]. Stages A and B patients were defined as those with risk factors that clearly predispose toward the development of heart failure [15]. Stage C denoted patients with current or past symptoms of heart failure associated with underlying structural heart disease [15]. Diabetes mellitus was defined as the recent use of antidiabetic drugs, a fasting blood glucose value of $\geq 126 \mathrm{mg} / \mathrm{dL}$ and/or a hemoglobin A1c value of $\geq 6.5 \%$. The exclusion criteria were patients with hyperglycemic crisis including diabetic ketoacidosis, lung cancer, and allergic asthma. Twenty patients with or at risk of heart failure (stage A or B heart failure) and diabetes mellitus were also enrolled (stage A or B group) [15]. The stage A or B group included 10 cases of angina pectoris, five cases of previous myocardial infarction, two cases of hypertension, two cases of atrial fibrillation, and one case of sick sinus syndrome. Baseline data of sex, age, body mass index, New York Heart Association class, etiology of heart failure, past medical history, physical findings, and current medication were collected at the time of enrollment in this study. This study protocol was approved by the Institutional Ethics Committee of Fukushima Medical University. Written informed consent was provided by all patients.

\section{Blood test and breath analysis}

All patients received a hospital diet with a median intake (interquartile range) of total energy, carbohydrate, protein, and fat was 1600 (1600-1800) kcal/day, 240 (235-263) g, 70 (66-78) g, and 41 (41-48) g, respectively. Blood samples were analyzed for estimated glomerular filtration rate (eGFR), total cholesterol, total bilirubin, glucose, hemoglobin A1c, brain natriuretic peptide, and total ketone bodies. Breath was collected for analysis once in the early morning after at least a 12-h fast at a stable phase. A breath-sampling bag (Collection Bag, Laboratory for Expiration Biochemistry, Nourishment Metabolism Co., Ltd., Nara, Japan) was used to maintain the breath. The collected breath was transferred to a gas-tight glass syringe, and $5 \mathrm{ml}$ was injected into a gas analysis device (GC-8A; Shimadzu Co., Ltd., Kyoto, Japan) to measure breath acetone concentration within the day of breath collection. Every measurement of the breath acetone was calibrated by standard gas (Sumitomo Seika Chemicals Co., LTD., Osaka, Japan). Acetone concentration in the breath of each patient was calculated by subtracting the acetone concentration of ambient air around the patients measured using the same method for the breath. The sampling method to evaluate exhaled acetone was previously reported and only the gas analysis device was different from the previous study [17].

\section{Echocardiography}

Echocardiography was performed by a blinded, experienced echocardiographer using the standard techniques [18]. The echocardiographic parameters investigated included left ventricular diastolic diameter, left ventricular systolic diameter, and left atrial diameter from the parasternal long-axis view. Left ventricular ejection fraction was measured by the modified biplane Simpson's method. Peak early (E) diastolic transmitral filling velocity and deceleration time of $\mathrm{E}$ diastolic transmitral filling velocity were measured.

\section{Statistical analysis}

Data were analyzed using the Statistical Package for Social Sciences version 24 (SPSS Inc., Chicago, IL, USA). All quantitative data are expressed as mean $\pm \mathrm{SD}$, or median and interquartile range. The statistical significance of differences was analyzed using Student's t-test for parametric continuous variables, and the Mann-Whitney U-test for nonparametric continuous variables. Categorical variables were compared using the Chi-square test or Fisher's exact test. Correlations were analyzed using Spearman's correlation analysis for variables. $P<0.05$ was considered statistically significant.

\section{Results}

Baseline characteristics of the study groups

In the baseline characteristics, the stage $C$ group had significantly higher levels of brain natriuretic peptide, larger left ventricular diameter and left atrial diameter, higher $\mathrm{E}$ diastolic transmitral filling velocity, and more frequent use of $\beta$-blocker, compared with the stage A or B group. The stage $\mathrm{C}$ group also had lower left ventricular ejection fraction, and lower levels of eGFR. The total ketone bodies in the blood, and the use of sodium-glucose linked transporter-2 inhibitor, which is associated with elevated blood ketone bodies, were not significantly different between the stage $\mathrm{A}$ or $\mathrm{B}$ group and stage $\mathrm{C}$ group (Table 1 ).

\section{Exhaled acetone concentration between stage a or B group and stage $\mathrm{C}$ group}

The stage $C$ group had higher exhaled acetone concentrations than those in the stage A or B group $(p=0.013$, 
Table 1 Baseline characteristics of the study subjects

\begin{tabular}{|c|c|c|c|}
\hline & Stage A or B $(n=20)$ & Stage C $(n=35)$ & $P$ value \\
\hline Age, years & $69 \pm 8$ & $72 \pm 20$ & 0.431 \\
\hline Male & $15(75)$ & $21(60)$ & 0.260 \\
\hline $\mathrm{BMI}, \mathrm{kg} / \mathrm{m}^{2}$ & $22 \pm 4$ & $21 \pm 5$ & 0.109 \\
\hline Smoking history & $16(80)$ & $19(54)$ & 0.057 \\
\hline NYHA class, I/II/III/IV & - & $3 / 28 / 4 / 0$ & - \\
\hline $\mathrm{SBP}, \mathrm{mmHg}$ & $131 \pm 19$ & $124 \pm 22$ & 0.161 \\
\hline Heart rate, beats/min & $71 \pm 16$ & $77 \pm 19$ & 0.077 \\
\hline \multicolumn{4}{|l|}{ Etiology } \\
\hline Ischemic & - & $9(26)$ & - \\
\hline Valvular & - & $3(9)$ & - \\
\hline Cardiomyopathy & - & $13(37)$ & - \\
\hline Others & - & $10(29)$ & - \\
\hline Hypertension & $13(65)$ & $26(74)$ & 0.466 \\
\hline COPD & $4(20)$ & $2(6)$ & 0.119 \\
\hline Atrial fibrillation & $3(15)$ & $11(31)$ & 0.178 \\
\hline \multicolumn{4}{|l|}{ Laboratory data } \\
\hline eGFR, $\mathrm{mL} / \mathrm{min} / 1.73 \mathrm{~m}^{2}$ & $65 \pm 18$ & $44 \pm 22$ & $<0.001$ \\
\hline Total cholesterol, mg/dL & $173 \pm 32$ & $163 \pm 46$ & 0.430 \\
\hline Total bilirubin, mg/dL & $0.9 \pm 0.3$ & $0.9 \pm 0.4$ & 0.562 \\
\hline Glucose, mg/dL & $125 \pm 24$ & $125 \pm 37$ & 0.403 \\
\hline Hemoglobin A1c, \% & $7.0 \pm 1.2$ & $6.7 \pm 0.7$ & 0.719 \\
\hline BNP, pg/mL (median, IQR) & $32(15-44)$ & $274(61-459)$ & $<0.001$ \\
\hline $\mathrm{TKB}, \mu \mathrm{mL} / \mathrm{L}$ (median, IQR) & $158(68-271)$ & $228(75-448)$ & 0.186 \\
\hline \multicolumn{4}{|l|}{ Echocardiography } \\
\hline LVDd, mm & $47 \pm 7$ & $52 \pm 10$ & 0.046 \\
\hline LVDs, mm & $31 \pm 7$ & $40 \pm 12$ & 0.008 \\
\hline LVEF, \% & $58 \pm 7$ & $44 \pm 16$ & 0.005 \\
\hline$L A D, m m$ & $37 \pm 6$ & $44 \pm 8$ & 0.009 \\
\hline $\mathrm{E}, \mathrm{cm} / \mathrm{s}$ & $0.6 \pm 0.1$ & $0.8 \pm 0.3$ & 0.032 \\
\hline Dct, msec & $223 \pm 54$ & $198 \pm 67$ & 0.105 \\
\hline \multicolumn{4}{|l|}{ Medication } \\
\hline ACE-I/ARB & $10(50)$ & $22(63)$ & 0.352 \\
\hline$\beta$-blocker & $7(35)$ & $24(69)$ & 0.016 \\
\hline Insulin & $5(25)$ & $8(23)$ & 0.553 \\
\hline Oral antidiabetic agents except SGLT-2 inhibitor & $12(60)$ & $16(46)$ & 0.308 \\
\hline SGLT-2 inhibitor & $1(5)$ & $3(9)$ & 0.537 \\
\hline
\end{tabular}

Values are mean $\pm \mathrm{SD}$ or $\mathrm{n}(\%)$, or median and IQR

$B M I$ body mass index, NYHA New York Heart Association, SBP systolic blood pressure, COPD chronic obstructive pulmonary disease, eGFR estimated glomerular filtration rate, $B N P$ brain natriuretic peptide, IQR interquartile range, TKB total ketone body, $L V D d$ left ventricular diastolic diameter, $L V D s$ left ventricular systolic diameter, $L V E F$ left ventricular ejection fraction, $L A D$ left atrial diameter, $E$ peak early diastolic transmitral filling velocity, Dct deceleration time, $A C E-I$ angiotensinconverting enzyme inhibitor, $A R B$ angiotensin-receptor blocker, SGLT sodium-glucose linked transporter

Fig. 1). The median exhaled acetone concentration (interquartile range) was $0.65 \mathrm{ppm}(0.47-0.96 \mathrm{ppm})$ and $0.96 \mathrm{ppm}(0.74-1.62 \mathrm{ppm})$ in the stage A or B group and the stage $\mathrm{C}$ group, respectively.

\section{Correlations of exhaled acetone concentration in all patients}

Exhaled acetone concentration was correlated with total ketone bodies $(r=0.588, p<0.001$, Fig. 2a) and brain 


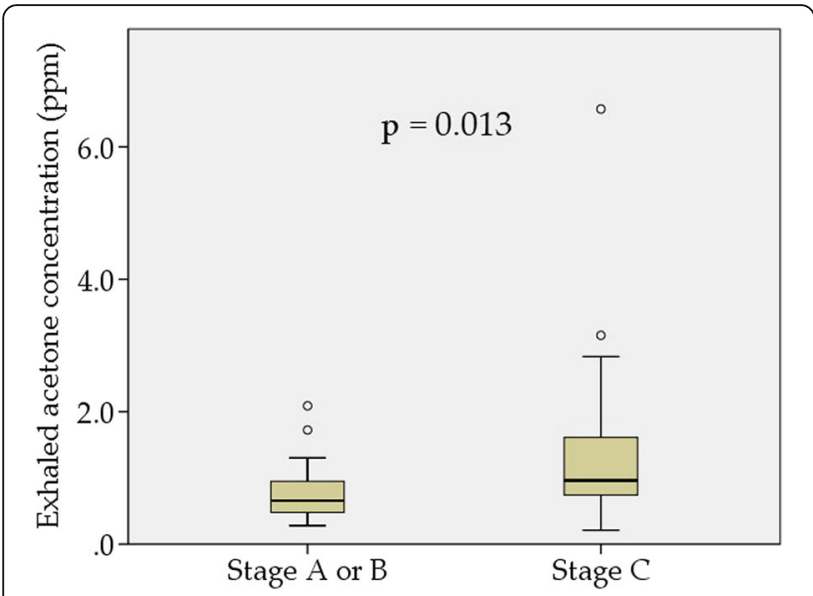

Fig. 1 Exhaled acetone concentration between the stage A or B group and the stage $C$ group. The stage $C$ group had higher exhaled acetone concentration than the stage $\mathrm{A}$ or $\mathrm{B}$ group $(p=0.013)$

natriuretic peptides $(r=0.415, p=0.002$, Fig. $2 b)$. On the other hand, exhaled acetone concentration was not correlated with hemoglobin A1c $(r=-0.035, p=0.801$, Fig. 3a) and eGFR ( $r=-0.073, p=0.597$, Fig. 3b).

\section{Discussion}

To the best of our knowledge, this is the first study to reveal that diabetic patients with stage $C$ heart failure have elevated exhaled acetone concentration, compared with diabetic patients with stage A or B heart failure. In addition, exhaled acetone concentration is correlated with heart failure severity in heart failure patients with diabetes mellitus.

Breath acetone concentration is reported to be a noninvasive biomarker of heart failure and is correlated with blood total ketone bodies, which are affected by diabetes mellitus [7, 8, 13, 19]. Many previous studies on breath acetone concentration and heart failure excluded the patients with diabetes mellitus in order to evaluate the direct relationship between breath acetone concentration and heart failure $[7,8,14,17]$. However, diabetes mellitus is a common comorbidity in patients with heart failure [12]. Breath acetone concentration should have been evaluated in heart failure patients with diabetes mellitus to establish the usefulness of exhaled acetone concentration as a biomarker of heart failure. This study demonstrates that exhaled acetone concentration is elevated in stage $\mathrm{C}$ heart failure patients with diabetes mellitus, and could be a noninvasive biomarker in heart failure patients with diabetes mellitus.

The relationship between diabetes mellitus and exhaled acetone concentration has been controversial with some studies reporting elevated exhaled acetone concentrations in diabetes mellitus [19-22]. Other reports state that there are no obvious differences between diabetic patients and non-diabetic patients with regard to exhaled acetone concentrations [23]. In the present study, there was no correlation between exhaled acetone concentration and hemoglobin A1c in heart failure patients with diabetes mellitus. According to the present study, the severity of diabetes mellitus might not be directly correlated with exhaled acetone concentration in heart failure patients with diabetes mellitus.

Breath markers of kidney disease was described in a previous study [24]. Kidney disease did not cause elevated breath acetone concentration [24]. In our study, eGFR was not correlated with exhaled acetone concentration. Although the stage $C$ group had both lower eGFR and elevated breath acetone concentration, the elevated acetone concentration was considered to be caused by heart failure.

Exhaled acetone concentration is affected by pulmonary congestion due to heart failure. We previously reported the correlation between exhaled acetone concentration and pulmonary capillary wedge pressure [17]. Increased pulmonary capillary hydrostatic pressure causes alveolar flooding and can increase the release of acetone into
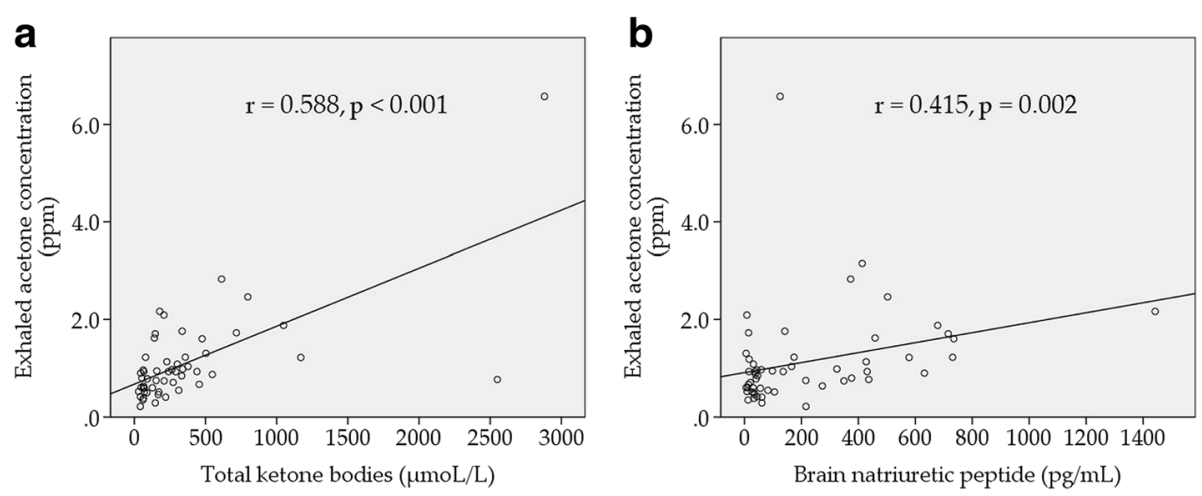

Fig. 2 Correlations of exhaled acetone concentration with total ketone bodies (a) and brain natriuretic peptide (b). Exhaled acetone concentration was correlated with total ketone bodies $(r=0.588, p<0.001)$ and brain natriuretic peptide $(r=0.415, p=0.002)$ 

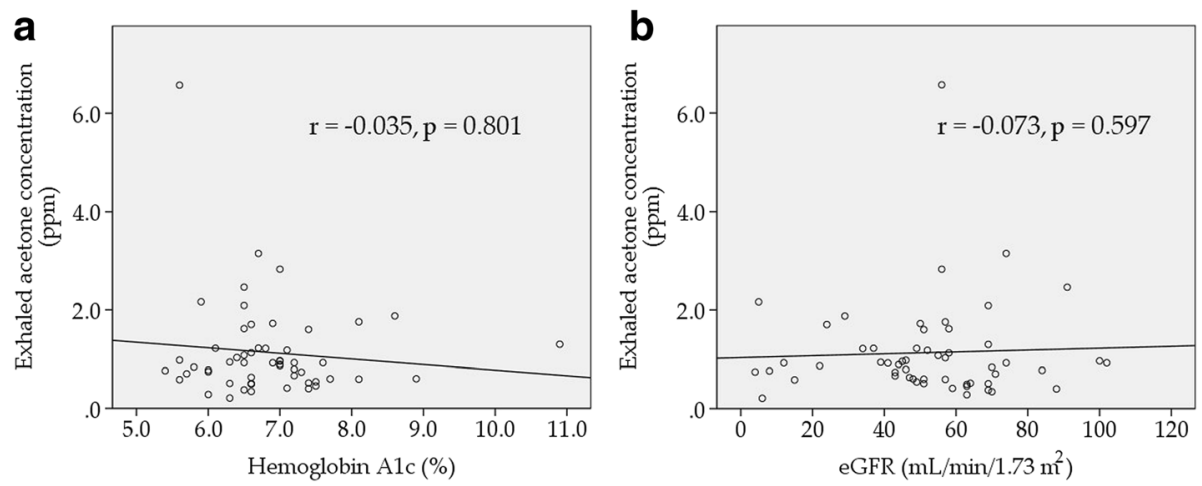

Fig. 3 Correlations of exhaled acetone concentration with hemoglobin A1c (a) and eGFR (b). Exhaled acetone concentration was not correlated with hemoglobin A1c and estimated glomerular filtration rate (eGFR)

airways. Pulmonary congestive state itself might increase exhaled acetone concentration in stage $C$ heart failure patients [17]. Exhaled acetone concentration could be a heart failure biomarker which reflects both blood ketone bodies and pulmonary congestion.

Among ketone bodies, acetone itself is correlated with elevated myocardial energy expenditure [5]. Elevated myocardial energy expenditure has been reported to be associated with reduced left ventricular ejection fraction and cardiovascular mortality [25]. Although the systemic and myocardial metabolism of ketone bodies remains unclear, detecting acetone might be a clue to understanding pathophysiological mechanisms in heart failure patients.

Measurement of exhaled acetone concentration is a noninvasive method, which is advantageous compared to the measurement of brain natriuretic peptide as it uses a needle for blood collection. Previous studies reported comparable diagnostic accuracy of exhaled acetone concentration with brain natriuretic peptide for heart failure in non-diabetic patients $[8,17]$.

A limitation of this study is that we used a small number of patients and a larger study population is required to evaluate exhaled acetone concentration as a biomarker of heart failure. Second, there were a lot of heart failure etiologies in the enrolled patients. The etiologies might have influenced the present results, and further study which evaluates the effects of exhaled acetone for heart failure etiologies with larger population is needed. Third, the correlation between exhaled acetone and blood total ketone bodies might be affected by other conditions. Exhaled acetone should be considered when diagnosing other conditions such as lung cancer, allergic asthma, and pulmonary congestion $[9,10,17]$.

\section{Conclusion}

Exhaled acetone concentration might be a noninvasive biomarker in heart failure patients with diabetes mellitus, correlating heart failure severity.
Abbreviations

E: Peak early; eGFR: Estimated glomerular filtration rate

\section{Acknowledgements}

Thank you to all of the members of the Department of Cardiovascular Division of Fukushima Medical University Hospital.

\section{Funding}

This work was supported by the Japanese Society for the Promotion of Science Kakenhi Grant No. 15 K19401 (T.Y.).

\section{Availability of data and materials}

The data that support the findings of this study are available at Fukushima Medical University Hospital but restrictions apply to the availability of these data, which were used under license for the current study, and so are not publicly available. Data are however available from the authors upon reasonable request and with permission of Fukushima Medical University Hospital.

\section{Authors' contributions}

Study design: TY, AS, and YT. Collection and interpretation of data: TS, SS, MO, AY, AK, TY, HK, KN, HS, SIS, and TI. Analysis of data: TY. Manuscript writing: TY and YT. All authors read and approved the final manuscript.

Ethics approval and consent to participate

This study protocol was approved by the Institutional Ethics Committee of Fukushima Medical University. Written informed consent was provided by all patients.

Consent for publication

Not applicable.

\section{Competing interests}

The authors declare that there are no competing interests.

\section{Publisher's Note}

Springer Nature remains neutral with regard to jurisdictional claims in published maps and institutional affiliations.

\section{Author details}

'Department of Cardiovascular Medicine, Fukushima Medical University, 1 Hikarigaoka, Fukushima, Fukushima 960-1295, Japan. ²Department of Lifelong Sports for Health Biochemical Sciences, College of Life and Health Sciences, Chubu University, Kasugai, Aichi, Japan. 
Received: 21 May 2017 Accepted: 9 November 2017

Published online: 16 November 2017

\section{References}

1. van Bilsen M, van Nieuwenhoven FA, van der Vusse GJ. Metabolic remodelling of the failing heart: beneficial or detrimental? Cardiovasc Res. 2009:81:420-8.

2. Aubert G, Martin OJ, Horton JL, Lai L, Vega RB, Leone TC, Koves T, Gardell SJ, Kruger M, Hoppel CL, Lewandowski ED, Crawford PA, Muoio DM, Kelly DP. The failing heart relies on Ketone bodies as a fuel. Circulation. 2016;133:698-705.

3. Bedi KC Jr, Snyder NW, Brandimarto J, Aziz M, Mesaros C, Worth AJ, Wang LL, Javaheri A, Blair IA, Margulies KB, Rame JE. Evidence for Intramyocardial disruption of lipid metabolism and increased myocardial Ketone utilization in advanced human heart failure. Circulation. 2016;133:706-16.

4. Janardhan A, Chen J, Crawford PA. Altered systemic ketone body metabolism in advanced heart failure. Tex Heart Inst J. 2011;38:533-8.

5. Du Z, Shen A, Huang Y, Su L, Lai W, Wang P, Xie Z, Xie Z, Zeng Q, Ren H, Xu D. 1H-NMR-based metabolic analysis of human serum reveals novel markers of myocardial energy expenditure in heart failure patients. PLoS One. 2014;9:e88102.

6. Lommi J, Kupari M, Koskinen P, Naveri H, Leinonen H, Pulkki K, Harkonen M. Blood ketone bodies in congestive heart failure. J Am Coll Cardiol. 1996;28:665-72.

7. Kupari M, Lommi J, Ventila M, Karjalainen U. Breath acetone in congestive heart failure. Am J Cardiol. 1995;76:1076-8.

8. Marcondes-Braga FG, Gutz IG, Batista GL, Saldiva PH, Ayub-Ferreira SM, Issa VS, Mangini S, Bocchi EA, Bacal F. Exhaled acetone as a new biomaker of heart failure severity. Chest. 2012;142:457-66.

9. Bassareo PP, Monte I, Romano C, Deidda M, Piras A, Cugusi L, Coppola C, Galletta F, Mercuro G. Cardiotoxicity from anthracycline and cardioprotection in paediatric cancer patients. J Cardiovasc Med (Hagerstown). 2016;17(Suppl 1):S55-63.

10. Fulcher $Y G$, Fotso M, Chang CH, Rindt H, Reinero CR, Van Doren SR. Noninvasive recognition and biomarkers of early allergic asthma in cats using multivariate statistical analysis of NMR spectra of exhaled breath condensate. PLoS One. 2016;11:e0164394.

11. Bajtarevic A, Ager C, Pienz M, Klieber M, Schwarz K, Ligor M, Ligor T, Filipiak W, Denz H, Fiegl M, Hilbe W, Weiss W, Lukas P, Jamnig H, Hackl M, et al. Noninvasive detection of lung cancer by analysis of exhaled breath. BMC Cancer. 2009:9:348.

12. Cavender MA, Steg PG, Smith SC Jr, Eagle K, Ohman EM, Goto S, Kuder J, Im K, Wilson PW, Bhatt DL. Impact of diabetes mellitus on hospitalization for heart failure, cardiovascular events, and death: outcomes at 4 years from the reduction of Atherothrombosis for continued health (REACH) registry. Circulation. 2015:132:923-31.

13. Avogaro A, Valerio A, Gnudi L, Maran A, Zolli M, Duner E, Riccio A, Del Prato S, Tiengo A, Nosadini R. Ketone body metabolism in NIDDM. Effect of sulfonylurea treatment. Diabetes. 1992;41:968-74.

14. Samara MA, Tang WH, Cikach F Jr, Gul Z, Tranchito L, Paschke KM, Viterna J, Wu Y, Laskowski D, Dweik RA. Single exhaled breath metabolomic analysis identifies unique breathprint in patients with acute decompensated heart failure. J Am Coll Cardiol. 2013;61:1463-4.

15. Jessup M, Abraham WT, Casey DE, Feldman AM, Francis GS, Ganiats TG, Konstam MA, Mancini DM, Rahko PS, Silver MA, Stevenson LW, Yancy CW. 2009 focused update: ACCF/AHA guidelines for the diagnosis and Management of Heart Failure in adults: a report of the American College of Cardiology Foundation/American Heart Association task force on practice guidelines: developed in collaboration with the International Society for Heart and Lung Transplantation. Circulation. 2009;119:1977-2016.

16. McKee PA, Castelli WP, McNamara PM, Kannel WB. The natural history of congestive heart failure: the Framingham study. N Engl J Med. 1971;285: 1441-6.

17. Yokokawa T, Sugano $Y$, Shimouchi A, Shibata A, Jinno N, Nagai T, Kanzaki H, Aiba T, Kusano K, Shirai M, Takeishi Y, Yasuda S, Ogawa H, Anzai T. Exhaled acetone concentration is related to hemodynamic severity in patients with non-ischemic chronic heart failure. Circ J. 2016;80:1178-86.

18. Lang RM, Badano LP, Mor-Avi V, Afilalo J, Armstrong A, Ernande L, Flachskampf FA, Foster E, Goldstein SA, Kuznetsova T, Lancellotti P, Muraru $\mathrm{D}$, Picard $\mathrm{MH}$, Rietzschel ER, Rudski L, et al. Recommendations for cardiac chamber quantification by echocardiography in adults: an update from the American Society of Echocardiography and the European Association of Cardiovascular Imaging. J Am Soc Echocardiogr. 2015;28:1-39.e14.
19. Wang Z, Wang C. Is breath acetone a biomarker of diabetes? A historical review on breath acetone measurements. J Breath Res. 2013;7:037109.

20. Henderson MJ, Karger BA, Wren Shall GA. Acetone in the breath; a study of acetone exhalation in diabetic and nondiabetic human subjects. Diabetes. 1952;1:188-93. passim

21. Tassopoulos CN, Barnett D, Fraser TR. Breath-acetone and blood-sugar measurements in diabetes. Lancet. 1969;1:1282-6.

22. Sun $M$, Chen Z, Gong Z, Zhao X, Jiang C, Yuan Y, Wang Z, Li Y, Wang C. Determination of breath acetone in 149 type 2 diabetic patients using a ringdown breath-acetone analyzer. Anal Bioanal Chem. 2015;407:1641-50.

23. Storer M, Dummer J, Lunt H, Scotter J, McCartin F, Cook J, Swanney M, Kendall D, Logan F, Epton M. Measurement of breath acetone concentrations by selected ion flow tube mass spectrometry in type 2 diabetes. J Breath Res. 2011;5:046011.

24. Grabowska-Polanowska B, Faber J, Skowron M, Miarka P, Pietrzycka A, Sliwka I, Amann A. Detection of potential chronic kidney disease markers in breath using gas chromatography with mass-spectral detection coupled with thermal desorption method. J Chromatogr A. 2013;1301:179-89.

25. Palmieri V, Roman MJ, Bella JN, Liu JE, Best LG, Lee ET, Howard BV, Devereux RB. Prognostic implications of relations of left ventricular systolic dysfunction with body composition and myocardial energy expenditure: the strong heart study. J Am Soc Echocardiogr. 2008;21:66-71.

\section{Submit your next manuscript to BioMed Central and we will help you at every step:}

- We accept pre-submission inquiries

- Our selector tool helps you to find the most relevant journal

- We provide round the clock customer support

- Convenient online submission

- Thorough peer review

- Inclusion in PubMed and all major indexing services

- Maximum visibility for your research

Submit your manuscript at www.biomedcentral.com/submit
) Biomed Central 